\title{
EN TORNO AL AÑO DE FUNDACIÓN DEL MONASTERIO DE VILLANUEVA DE LORENZANA
}

\author{
por' \\ MANUEL CARRIEDO TEJEDO
}

El documento de fundación del monasterio de Villanueva de Lorenzana, expedido por el conde Osorio Gutiérrez un "die quod erit quintodecimo kalendas iulias, era septima post millesima", esto es, el 17 de junio de 969 , presenta importantes inconvenientes en el año de la data, como consecuencia de la presencia incompatible, cronológicamente hablando, de varios de los siete obispos que se mencionan, tanto en el texto de la escritura como en el escatocolo:

\footnotetext{
- Episcopus Domini Thoemirus sedis $\quad$ - Theodemirus episcopus

Minduniensis

- Ermigildus Bracharensis episcopus - Ermegildus episcopus

- Rudesindus episcopus Dumiensis

- Rudesindus episcopus Dumiense sedis Cellenovense

- Gundisalvus episcopus Legionensis

- Gundesiobbus episcopus Legionense

- Sisnandus episcopus Iriense sedis

- Sisnandus episcopus

- Villiulfus episcopus (de Tuy)

- Villulfus episcopus

- Rudericus episcopus

- Rudericus episcopus
}

En efecto, descartados los largos pontificados de Hermenegildo de Lugo

\footnotetext{
${ }^{1}$ Ed. H. FLÓREZ, España Sagrada (=ES), XVIII, 332-340. 
(951-985) y Viliulfo de Tuy (951-1002) $)^{3}$, que exceden muy sobradamente la cronología en estudio, la datación de la escritura laurentina en 969 presenta dos graves anacronismos que afectan, en mayor o menor grado, a los otros cinco prelados que se mencionan:

- Porque según una escritura leonesa, de 1 de septiembre de 966, el titular de esta diócesis era "Gundisalbus, Dei gratia aepiscopus"4, y por otro documento también leonés y original, fechado el 4 de octubre de 968 , sabemos que ya le había sucedido "Belascus, Dei gratia, aepiscopus Legionense sedis", que suscribe junto al entonces titular de Mondoñedo, "Rudericus, Dei gratia, aepiscopus Dumiense sedis"5.

- Porque Sampiro es muy claro al fijar la muerte de Sisnando de Santiago en el segundo año del cómputo de Ramiro III, a manos de los viquingos: "Anno secundo regni sui, centum classes normanorum cum rege suo Gunderedo, ingresse sunt urbes Gallecie, et strages multas facientes in giro sancti Iacobi, episcopum loci illius gladio peruenerunt nomine Sisinandum"6. Y es sabido que la última carta conocida de Sancho I es de 15 de noviembre de $966^{7}$; que en 966 , antes del 31 de diciembre,

${ }^{2}$ Confirma su primera escritura, junto a su homónimo de Santiago, el 5 de marzo de 951 (ed. A. LÓPEZ FERREIRO, Historia de la Santa A.M. Iglesia de Santiago de Compostela, II, Santiage 1899, doc. 61, 136-138); y desaparece de los documentos el 29 de septeimbre de 985 (Tumbo de Celanova, f. 174 r.; ed. L. BARRAU-DIHIGO, Notes et documents sur l'histoire du royaume de Leon. I. Chartes royales léonaises: Revue Hispanique, X, 1903, doc. 30, 423-426) (=BARRAU-DIHIGO, Chartes royales léonaises).

${ }^{3}$ La primera mención segura de Viliulfo en una carta corresponde también a la de 5 de marzo de 951 (ed. A. LÓPEZ FERREIRO, Historia de Santiago, doc. 61, 136-138); y la última está fechada el 21 de diciembre de 1002 (ed. J.M. RUIZ ASENCIO, Colección documental del Archivo de la catedral de León (775-1230), III (986-1031), León 1987, doc. 629, 162-165 (Colección: "Fuentes y Estudios de Historia Leonesa"; núm. 43).

${ }^{4}$ Ed. E. SAEZ y C. SAEZ, Colección documental del Archivo de la catedral de León (775-1230), II (953-985), León 1990, doc. 397, 182 (Colección: "Fuentes y Estudios de Historia Leonesa"; núm. 42) (=SAEZ, Colección catedral de León, II).

${ }^{5}$ ACL núm. 909; ed. ID., ibid., doc. 410, 198-199. Con anterioridad a este original, ambos aparecen también en otra carta de Sobrado de 17 de septiembre de 968 (ed. P. LOSCERTALES DE G. VALDEAVELLANO, Tumbos del monasterio de Sobrado de los Monjes, I, Madrid 1976, doc. 107, 127-130) (=LOSCERTALES, Tumbos de Sobrado, I).

${ }^{6}$ Crónica de Sampiro; ed. J. PÉREZ DE URBEL, Sampiro: Su crónica y la monarquía leonesa en el siglo X, Madrid 1952, 340 (=PÉREZ DE URBEL, Sampiro). 58.

${ }^{7}$ Portugaliae Monumenta Historica, I. Diplomata et Chartae, Lisboa 1867, doc. 92,

"CUADERNOS DE ESTUdios GALLEGOS", Tomo XLI, Fascículo 106, Santiago 1993-94. 
fechan su muerte la "Historia Silense"8 y la "Crónica Najerense"9; que Sampiro sitúa en 967 el comienzo de su hijo Ramiro III ${ }^{10}$; y que el primer documento original que cita a este monarca es de 27 de marzo de $967^{11}$. Así pues, el segundo año de Ramiro III hubo de ser el 968, y el 17 de septiembre de dicho año Sisnando ya había muerto, según una carta de Sobrado ${ }^{12}$, felizmente sincrónica con el día exacto señalado por la "Historia Compostelana", 29 de marzo de 968: "Occisus est IIII. K1. Aprilis Era I. $\mathrm{VI}^{\prime \prime}$.

${ }^{8}$ Historia Silense: "Era igitur millesima quarta, defuncto Sancio rege..." (ed. PÉREZ DE URBEL y A. GONZÁLEZ RUIZ ZORRILA, Madrid 1959, 173).

${ }^{9}$ Crónica Najerense: "Regnauit annos XII, et obiit era MIIII" (ed. A. UBIETO ARTETA, Valencia 1966, 79).

${ }^{10}$ Crónica de Sampiro: "Era MV. Sancio defuncto, filius eius Ramirus habens a natiuitate annos quinque suscepit regnum patris sui" (ed. PÉREZ DE URBELM, Sampiro, 339).

${ }^{1}$ ACL, núm. 840; ed. SÁEZ, Colección catedral de León, II, doc. 401, 186-187: "Regnanti Ranemiro infans, anno primo regni sui".

12 "Predecessores uestros Hermenegildus et Paterna, Sisnandus episcopus et suus germanus Rudericus usque finem eorum... Facta serie testamenti XV kalendas octobris, era millesima VI in ciuitate Legionense" (ed. LOSCERTALES, Tumbos de Sobrado, I, doc. 107, 127-130).

${ }^{13}$ Historia Compostelana; ed. FLÓREZ, ES, XX, 13. Para López Ferreiro (Historia de Santiago, II, 355) "el Concilio pudo celebrarse en el año 967, y de este modo no hay inconveniente en que a él asistiese Sisnando"; porque este autor sí reparó en la escritura de Sobrado de 17 de septiembre de 968, que da por muerto a Sisnando (nota 12). Sin embargo, Sáez (Notas al episcopologio minduniense del siglo X: Hispania, VI, 1946, 36-37, nota 137) insiste en que la escritura fundacional de Lorenzana "no es posible esté errada", rechaza, de forma acertada, el año 967 propuesto por López Ferreiro, en base a la carta de 4 de octubre de 968, que presenta como obispo de Mondoñedo a Rodrigo (ed. ID., Colección catedral de León, II, doc. 410, 198-199), y concluye aduciendo un posible yerro en la fecha de la escritura de Sobrado, que "puede ser de 969 en vez de 968". Y para García Álvarez (Sobre el episcopado de Viliulfo en Tuy: El Museo de Pontevedra, IX, 1955, 71 72 , nota 38 y 77) que como los anteriores tampoco reparó en el testimonio de Sampiro, el obispo Sisnando murió entre el 17 de junio de 969, fecha de la fundación de Lorenzana, que también da por buena, y el 17 de septiembre de 969, año propuesto por Sáez para la carta de Sobrado, que de igual modo asume. El mismo García Álvarez, que afrontó años después el estudio del obispado de Sisnando II de Santiago (Sisnando Menéndez, mayordomo real y obispo de Santiago: Compostellanum, XIII, 2, 1968, 235-239), concluye diciendo que "como la escritura de Lorenzana sólo puede ser del año 969 y como en ella aparecen dos prelados, Sisnando de Iria y Gonzalo de León, que no lo eran ciertamente en dicho año, no queda más solución que suponer interpolada tal escritura... en definitiva, creo poder afirmar sin recelo que... más que un documento diplomático es un texto religioso, no siendo lícito esgrimirlo como argumento para retrasar la muerte de Sisnando hasta el año 970. Sisnando murió, pues, el 29 de marzo de 968 ... que era el segundo del reinado de Ramiro III".

"CUADERNOS DE ESTUDIOS GALLEGOS", Tomo XLI, Fascículo 106, Santiago 1993-94. 
- Porque muerto Sisnando Menéndez se sentó en la silla compostelana el que era entonces abad de Celanova y obispo dimisionario de Mondoñedo, San Rosendo ${ }^{14}$, según declaran al unísono: 1) El "Chronicon Iriense": "Sisnandus iam diaconatus ordine functus, Menendi Comitis filius, in ordine in loco sancto VII consecratur episcopus... Rodesindus in sede Apostolica VIII levatur episcopus"15; 2) La "Historia Compostelana": "Reodosindum Cellenove religiosum monacum eius in honorem subrogavit"16; 3) Y los documentos, entre los cuales uno original de 29 de julio de 974, "Rudesindus Dei gratia episcopus Iriense sedis", que también confirma el sucesor de Rodrigo en la sede Mondoñedo, "Theodomirus Dumiense sedis aepiscopus"17.

La consecuencia es clara, el día 17 de junio de 969, ni Gonzalo era obispo de León, pues ya le había sustituido Velasco en 968; ni Sisnando lo era de Santiago, porque murió el 29 de marzo de 968; ni San Rosendo era dimisionario de Mondoñedo, pues, muy probablemente, ya había sucedido en Santiago a Sisnando ${ }^{18}$.

Así pues, a la vista de cuanto antecede y con el resto de datos disponibles, que consignamos a continuación, se hace en verdad muy difícil situar la carta fundacional de Lorenzana en 969:

${ }^{14}$ Flórez, que no vio dificultades en el año 969 de la carta de Lorenzana, sitúa el pontificado de San Rosendo en Santiago "después de 970 y hasta el 977" (ES, XIX, 161).

${ }^{15}$ Chronicon Iriense; ed. FLÓREZ, ES, XIV, 604-605.

${ }^{16}$ Historia Compostelana; ed. FLÓREZ, ES, XX, 13.

${ }^{17}$ Ed. C. SÁNCHEZ-ALBORNOZ, El obispado de Simancas: Miscelánea de Estudios Históricos, León 1970, 392-396 (Colección: "Fuentes y Estudios de Historia Leonesa", núm. 3).

${ }^{18}$ En una donación a Celanova, del año 973, se dice: "Ideo offero uel concedo perpetualiter post parte monasterii uel pontifice domno Rudesindo episcopo uel fratribus uestris in ipso loco pie uiuentibus, uilla quam uocitant Sancto Tyrso" (Ed. SÁEZ, Notas, 75); frase que no excluye, necesariamente, su titularidad en la diócesis compostelana. Hay más ejemplos a este respecto en otros obispos que antes habían sido abades. El 1 de abril de 969, en pleno pontificado de Novidio de Astorga, se hace una donación a Samos: "Vobis domno Novidio episcopo et fratribus habitantibus in monasterii Samanos" (ed. M. LUCAS ÁLVAREZ, El Tumbo de San Julián de Samos (siglos VII-XII). Estudio introductorio, edición diplomática, apéndices e indices, Santiago de Compostela 1986, doc. 241, 425426) (=LUCAS ÁLVAREZ, Tumbo de Samos).

"CUADERNOS DE ESTUDIOS GALLEGOS", Tomo XLI, Fascículo 106, Santiago 1993-94. 


\begin{tabular}{lccccc} 
Documentos & ASTORGA & LEÓN & SANTIAGO & MONDOÑEDO & $\begin{array}{r}\text { DIMISIONARIOS } \\
\text { DE MONDOÑEDO }\end{array}$ \\
\hline $966-09-01^{19}$ & & & & & - \\
$966-12-10^{20}$ & - & Gonzalo & - & - & - \\
$968-03-29^{21}$ & - & - & Sisnando & - & - \\
$968-01-01^{22}$ & Novidio & Velasco & - & - & Rosendo \\
$968-09-17^{23}$ & - & - & + Sisnando & - & - \\
(id.) & Novidio & Velasco & Rosendo & Rodrigo & - \\
$968-10-04^{24}$ & Novidio & Velasco & Rosendo & Rodrigo & - \\
$969-02-19^{25}$ & - & Velasco & - & - & - \\
$969-04-01^{26}$ & Novidio & - & - & - & - \\
$969-06-17$ & - & (Gonzalo) & (Sisnando) & (Teodomiro) & (Rosendo/Rodrigo) \\
--- & & & & & - \\
$974-07-299^{27}$ & Gonzalo & Sisnando & Rosendo & Teodomiro & -
\end{tabular}

19 "Facta kartula uendiccionis die kalendas septembris, era MIIII... Gundisaluus, Dei gratia episcopus, conf." (ed. SÁEZ, Colección catedral de León, II, doc. 397, 182).

20 "Facta scriptura testamenti uel benefacti nostri IIII idus decembris, era MIIII... Sisnandus Dei gratia episcopus in hoc testamentum uel benefactum quod fieri elegi manu propria confirmo" (ed. LOSCERTALES, Tumbos de Sobrado, I, doc. 6, 33-37).

${ }^{21}$ Historia Compostelana: "Occissus est IIII. Kl. Aprilis Era I. VI"; ed. FLÓREZ, ES, $\mathrm{XX}, 13$.

${ }^{22}$ Ed. BARRAU-DIHIGO, Chartes royales léonaises, doc. 18, 390-392.

23 "Hermenegildus et Paterna, Sisnandus episcopus et suus germanus Rudericus usque finem eorum... Facta serie testamenti XV kalendas octobris, era millesima VI in ciuitate Legionense... Rudesindus Dei gratia episcopus... Uiliulfus Tudensis episcopus... Rudericus episcopus... Nouidius episcopus... Uelascus episcopus". (ed. LOSCERTALES, Tumbos de Sobrado, I, doc. 107, 127-130).

${ }^{24}$ ACL núm. 909: "Rudesindus, Dei gratia aepiscopus, conf... Rudericus, Dei gratia, aepiscopus Dumiense sedis conf... Nouidius, Dei gratia, aepiscopus Astoricense sedis, conf... Belascus, Dei gratia, aepiscopus Legionense sedis" (ed. SÁEZ, Colección catedral de León, II, doc. 410, 198-199).

25 "Nodum die XI kalendas marcii, era MVII... Uelascus, Dei gratia, episcopus Leionense sedis" (ed. ID., ibid. doc. 411, 199-201).

26 "Ego Frogildi, vobis domno Novidio episcopo et fratribus hatitantibus in monasterii Samanos... Facta carta venditionis kalendis Aprilis, era MVII" (ed. M. LUCAS ÁLVAREZ, Tumbo de Samos, doc. 241, 425-426).

${ }^{27}$ Ed. SÁNCHEZ-ALBORNOZ, El obispado de Simancas, 392-396.

"CUADERNOS DE ESTUDIOS GALLEGOS", Tomo XLI, Fascículo 106, Santiago 1993-94. 
Las contradicciones son evidentes, y habrá que esperar al menos un lustro, según se acaba de anotar, para que los nombres (que no las sedes) de los dos obispos extemporáneos registrados en la escritura de Villanueva, Sisnando y Gonzalo, coincidan en otra carta con San Rosendo y con Teodomiro de Mondoñedo. ¿Qué pudo ocurrir para que la escritura fundacional de Lorenzana incurra en tan importantes contradicciones?

Aceptando que los nombres conservados en la carta sean los correctos, lo que parece avalar el hecho de que los siete obispos sean puntualmente recogidos tanto en el texto como en el escatocolo, cabría admitir, a modo de explicación, que fuera un copista del diploma el que añadió de su particular erudición, y de forma tan incompleta como confusa, unas sedes que tal vez el original silenció:

1) Porque, muerto Sisnando de Santiago en 968, es sabido que hubo poco tiempo después otro obispo Sisnando que pontificó en León entre 973 y $982^{28}$.

2) Porque, de igual modo, después de Gonzalo de León, muerto en 968, gobernó la diócesis de Astorga otro Gonzalo entre los años 973 y $992^{29}$.

El protagonismo del obispo Teodomiro de Mondoñedo (972-977) en el acto fundacional de Villanueva de Lorenzana está fuera de duda, es mencionado varias veces y en consecuencia parece difícil pensar en una posi-

${ }^{28}$ Su primera carta es de 23 de octubre de 973 : "Sisinandus, Legionense sedis episcopus, conf." (ed. SÁEZ, Colección catedral de León, II, doc. 425, 217-218). Y la última conocida, en cuya virtud recibe una donación de Ramiro III, aparece fechada el 14 de enero de 979: "Ranimirus, nutu diuino princeps, necnon et Santie regine... pontifice nostro domno Sisinando, Dei gratia episcopo... Notum die XVIIII kalendas februarii, era XVII post millesima. Regnante in regna paterna annis et imperii terquini sedis Legione, solium Ouetense" (ed. ID, ibid., doc. 482, 287-289); aunque su editor se decide por situarla en 981, atendiendo al decimoquinto año del cómputo regio. Criterio concordante, además, con el testimonio de otra escritura original leonesa de 27 de marzo de 967: "Regnanti Ranemiro infans, anno primo regni sui" (ACL, núm. 840; ed. ID., ibid., doc. 401, 186187).

${ }^{29}$ También aparece suscribiendo la carta de 23 de octubre de 973: "Gundisaluus, Astoricense sedis episcopus, conf." (ed. SÁEZ, Colección catedral de León, II, doc. 425, 217-218); y el 23 de enero de 992 todavía se encuentra otorgando una donación en su territorio diócesano: "Gundisalvus, Dei gratia episcopus, in hoc testamentum manu mea hoc signo eiecimus" (ed. A. QUINTANA PRIETO, El obispado de Astorga en los siglos IX $y$ X, Astorga 1968, 467).

"CUADERNOS DE ESTUDIOS GALLEGOS", Tomo XLI, Fascículo 106, Santiago 1993-94. 
ble confusión o error en la transmisión manuscrita: "Unde initium aedificii cenobium locutus fui cum episcopo domini Thoemirus, postulavi ab eo consilium, qualiter pervenirem ad augendam norman sancti Benedicti et quomodo haberent ab ipso episcopo una cum aliis provincialibus concedentibus discretionem sanctam et justam secundum canones Esidorus Hispalensis... Hinc inde omnibus episcopis ad invicem conloquentibus, nominibus id sunt: Thoemirus, sede Minduniensis... Unde ausus respondit episcopus domini Thoemirus sede Minduniensi, una cum consensu episcoporum, seu concilio omnis statuti confirmantes... Sub Christi nomine Theodemirus episcopus confirmat" ${ }^{30}$.

Y no puede extrañar que en tal acontecimiento le acompañaran, nunca de forma tan justificada por tratarse de un acto relacionado con la propia diócesis minduniense, los otros dos prelados dimisionarios que le habían precedido, San Rosendo y Rodrigo, a los que quizá no supo identificar el copista de forma satisfactoria en la escritura:

3) Porque de haber conocido el muy corto pontificado de Rodrigo en Mondoñedo (968-970), no habría dejado de consignarlo en el texto o en la confirmación, visto el supuesto que defendemos ${ }^{31}$.

${ }^{30}$ Ed. FLÓREZ, ES, XVIII, 332-340. El primer documento conocido de Teodomiro es de 11 de agosto de 972; cit. ID., Notas, 38, nota 140; reg. GARCÍA ÁLVAREZ, Catálogo de documentos reales de la Alta Edad Media referentes a Galicia: Compostellanum, VIII, 1963, doc. 351, 288-289 (=GARCÍA ÁLVAREZ, Catálogo); y el último de 16 de junio de 977; ed. J.M. MINGUEZ, Colección diplomática del monasterio de Sahagún (siglos IXy X), I, León 1976, doc. 290, 349-351 (Colección "Fuentes y Estudios de Historia Leonesa", núm. 17).

${ }^{31}$ Cree Sáez posible (Notas, 30 y 34) que el "Rudericus episcopus" que suscribe en primer lugar la carta de Ordoño IV a Compostela de 21 de mayo de 958 (ed. LÓPEZ FERREIRO, Historia de Santiago, II, doc. 71, 165-166), sustituyera a Arias en Mondoñedo durante tan corto reinado. Aunque tal vez el copista del Tumbo A escribió "Rudericus" por "Rudesindus", obispo que sí aparece, el primero, en otras donaciones de Ordoño IV a Santiago de 2 de marzo de 958 (ID., ibid. docs. 69 y 70, 161-164). Y es de advertir que otro "Rudericus, confessor, nutu divino prontificali gratia constitutus", aparece junto a Odoario de Astorga el 28 de junio de 959 (ed. QUINTANA PRIETO, Temas Bercianos, I, 467-468). Por ello, parece más fácil pensar en San Rosendo como sustituto de Arias entre 955 y 959 (vid. SÁEZ, ibid., 29-30), y no el incógnito Rodrigo de 958 y 959, no identificable con el homónimo que un decenio después obtuvo la silla minduniense, según una carta original de 4 de octubre de 968: "Rudericus, Dei gratia, aepiscopus Dumiense sedis conf." (ed. ID., Colección catedral de León, II, doc. 410, 198-199). En cuanto a los diplomas del Tumbo de León, de 29 de enero y 23 de junio de 970, que citan a "Rudericus, Legionense sedis episcopus", ambos son del mismo fondo, el de Santiago de León, los

"CUADERNOS DE ESTUDIOS GALLEGOS", Tomo XLI, Fascículo 106, Santiago 1993-94. 
4) Porque el tal copista minduniense sí pudo estar informado, a lo que parece, sobre el largo pontificado de San Rosendo en Mondoñedo (927$950)^{32}$, y sobre su gobierno en el monasterio de Celanova, que es lo que consignó, utilizando una extraña fórmula, como ya observó en su día Sáez: "Rudesindus episcopus Dumiense sedis Cellenovense ${ }^{33}$; y ello por haber desconocido, muy probablemente, su segundo mandato al frente de la diócesis de Santiago (968-976) $)^{34}$.

confirmantes del segundo coinciden con los del primero, y los dos fueron copiados casi seguidos (ff. 334r-335r y 341 r-v; ed. ID., ibid, docs. 412 y 413, 201-204); y por ello, el copista pudo haber omitido, por error el salto de línea, la mención de Velasco, titular leonés: "Sub Christi nomine, Rudericus; (episcopus Dumiense sedis, confirmat. Sub diuina clementia, Belascus), Legionense sedis episcopus, confirmat". Sino, habrá que aceptar como posible, ignoramos debido a qué causas, la interinidad de Rodrigo de León (vid. ID., Notas, 36, nota 137), pero como máximo entre 19 de febrero de 969 y 12 de noviembre de 971 , fechas de las cartas respectivas que interrumpen y reanudan las menciones de Velasco (ed. ID., Colección catedral de León, II, docs. 411 y 415, 199-200 y 206-207). Si así fue, tampoco hay razones para dudar que Rodrigo siguiera, al menos enre 968 y 970 , al frente de Mondoñedo. Su sucesor Teodomiro no aparecerá hasta 11 de agosto de 972 (cit. ID., Notas, 38, nota 140; reg. GARCÍA ÁLVAREZ, Catálogo, doc. 351, 288-289.

${ }^{32}$ Creemos posible que el pontificado de San Rosendo en Mondoñedo no finalizará entre 944 y 948, como propone Sáez (Notas, 6-19), que presenta a su sucesor, Arias, en una carta de 25 de mayo que trae a cuento la "era DCCCCXXXI", año 898, pero que él sitúa en 948, al suponer omitida una "L" (cit. ID., ibid., 26, nota 107), y a la que también pueden faltarle una o tres "I" finales. Además, hay que tener en cuenta el diploma portugués de 18 de mayo de 951, expedido por Ramiro II, en el que está ausente el propio Arias, que sin embargo suscribe "Rudesindus Dumiensis episcopus"; y que el propio Sáez sitúa (ibid., 17, nota 67) en el año 950, creemos que con buen criterio.

${ }^{33}$ Dice Sáez (ibid., 18-19): "En la escritura de fundación del monasterio de Villanueva de Lorenzana, de 19 de junio de 969, figura la firma de San Rosendo de este extraño modo... aún suponiendo que el copista no haya alterado la suscripción primitiva, el título de 'Dumiense', que, como dice Flórez (ES, XVIII, 100), pudo emplear para indicar el sitio donde se consagró, está modificado por el de 'Cellenovense', y que indica la residencia... los adjetivos 'dumiense' y 'minduniense' tiene aquí el mismo significado y vemos que a partir de Teodomiro se utilizan indistintamente, hasta que acaba por quedar el último".

${ }^{34}$ Después de muerto Sisnando el 29 de marzo de 968, encontramos a San Rosendo, por ejemplo, en cartas de 17 de septiembre de 968: "Rudesindus Dei gratia episcopus cf." (ed. LOSCERTALES, Tumbos de Sobrado, I, doc. 107, 127-130); 4 de octubre de 968: "Rudesindus, Dei gratia aepiscopus, conf." (ed. SÁEZ, Colección catedral de León, II, doc. 410, 198-199); 973: "Pontifice domno Rudesindo episcopo", (ed. SÁEZ, Notas, 75); 29 de julio de 974: "Rudesindus Dei gratia episcopus Iriense sedis" (ed. SÁNCHEZ-ALBORNOZ, El obispado de Simancas, 392-396); y 3 de junio de 976: "Rudesindus episcopus confirmo" (ed. M. LUCAS ÁlVAREZ, Tumbo de Samos, doc. 61, 175-178).

"CUADERNOS DE ESTUDiOS GALLEGOS", Tomo XLI, Fascículo 106, Santiago 1993-94. 
Visto cuando antecede, y a modo de conclusión, el documento de Lorenzana, datado un día 17 de junio, pudo haberse expedido:

1) Con posterioridad al 11 de agosto de 972 , fecha del último diploma hoy conocido del obispo Novidio ${ }^{35}$, inmediato antecesor de Gonzalo de Astorga, aquí identificado con el "Gundisalvus" que suscribió el documento de Lorenzana.

2) Con anterioridad al mes de mayo de 977 , fecha de la primera carta llegada hasta hoy que menciona el pontificado compostelano de Pelayo Rodríguez, sucesor de San Rosendo ${ }^{36}$. En sincronía con la donación de Ramiro III a favor de Celanova, fechada el 21 de marzo de 977, que ya declara la reciente muerte del santo: "Monasterium situm est loco predicto Cellenoue, quód uenerabilis pater et diue memorie auius noster domnus Rudesindus episcopus fundauit et ubi sanctissimum corpus eius honorifice humatum abetur" ${ }^{137}$.

De todo lo dicho se obtendrían unos límites anuales posibles para la carta laurentina comprendidos entre 973 y 976, ambos inclusive, período en el que en efecto pontificaron todos los prelados cuyos nombres reproduce la escritura fundacional: "Rudesindus episcopus Dumiensis (por Iriensis); Ermigildus Bracharensis episcopus (con sede en Lugo); Domni Theodemirus, sede Minduniensis; Gundisalvus episcopus Legionensis (por Astoricensis); Sisnandus episcopus Iriense (por Legionensis) sedis; Villiulfus episcopus (que lo fue de Tuy); y Rudericus episcopus (a la sazón dimisionario de Mondoñedo)"38.

${ }^{35}$ Ed. M. SERRANO Y SANZ, Documentos del Cartulario del Monasterio de Celanova: Boletín de la Biblioteca Menéndez y Pelayo, III, 1921, doc. 7, 302-304. Cit. SÁEZ, Notas, 38, nota 140; reg. GARCÍA ÁLVAREZ, Catálogo, doc. 351, 288-289.

36 "Pelagius, episcopus Hyriense, conf." (ed. SÁEZ, Colección catedral de León, II, doc. 451, 253-255).

${ }^{37}$ Ed. BARRAU-DIHIGO, Chartes royales léonaises, doc. 25, 411-412.

${ }^{38}$ EI documento de Lorenzana aparece suscrito asimismo, y entre otros muchos, por el diácono "Scemenus Troctiniz", que se documenta junto al obispo de Sisnando de León en una escritura leonesa de Abellar de 24 de noviembre de 976: "Sisnandus, Dei gratia episcopus, conf... Scemenus, diachonus, Tructiniz, frater, notuit" (ed. SÁEZ, Colección catedral de León, II, doc. 446, 248-249). Pero además, es curioso observar que entrambos existía una estrecha relación de parentesco, según una carta samonense de 983: "Nos exigui et personaii, Scemenus archidiaconus filius Tructini, necnon et Uniscus, confesus, seu et Sandinus Baroncelli... Dubium quidem non est sed plerisque cognitum quod tius noster domnus Sisnandus episcopus, regens cathedram sedis legionense..." (ed. M. LUCAS ÁlVAREZ, Tumbo de Samos, doc. 156, 323-324).

"CUADERNOS DE ESTUdIOS GALLEGOS", Tomo XLI, Fascículo 106, Santiago 1993-94. 
Y ahí está sino el documento original de supresión de la sede de Simancas, fechado el 29 de julio de 974 y confirmado, junto a Ramiro III y el obispo Diego de Orense, por nada menos que cinco de los siete prelados presentes en Lorenzana, mencionados todos, ahora sí, al frente de sus sedes respectivas: "Rudesindus Dei gratia episcopus Iriense sedis; Ermegildus Lucense sedis episcopus confirmans; Theodemirus Dumiense sedis episcopus confirmans; Gundisalbus Astoricense sedis episcopus confrimans; y Sisinandus Dei gratia Legionense sedis episcopus confirmans"39.

${ }^{39}$ Ed. SÁNCHEZ-ALBORNOZ, El obispado de Simancas, 392-396. 